\title{
An Imaging System for Focusing Tests of Li Multiprism X-ray Refractive Lenses
}

\author{
E.M. Dufresne ${ }^{* \dagger}$, D.A. Arms ${ }^{\dagger}$, N.R. Pereira**, P. Ilinski ${ }^{\ddagger}$ and R. Clarke* \\ *Department of Physics, University of Michigan, Ann Arbor MI 48109 \\ ${ }^{\dagger}$ MHATT-CAT, Advanced Photon Source, Argonne National Lab., Argonne IL 60439 \\ ${ }^{* *}$ Ecopulse Inc., Springfield VA 22150 \\ ${ }^{*}$ Advanced Photon Source, Argonne National Lab., Argonne IL 60439
}

\begin{abstract}
For rapid and efficient tests of novel X-rays optics, such as lithium-based compound refractive lenses [1], we have built a fast X-ray sensitive CCD imaging system. We report on the linearity, response and resolution of the microscopebased imaging system. For the low magnifications used here (X2-X10), we find that a thinly doped YAG screen has a poorer resolution than a thick YAG screen. We provide an example of its use in testing a new 2D focusing multiprism X-ray lens.
\end{abstract}

Novel X-ray focusing optics, such as compound refractive lenses (CRL) [2] require a fast and high-resolution imaging system to characterize their optical properties. A standard imaging system for this type of application was described and tested in details several years ago [3]. Many groups around the world now use similar systems based on imaging the visible fluorescence of a thinly doped YAG:Ce crystal with a high-magnification microscope. Few have published detailed characterization of their imaging system[3]. We have designed a similar system to test novel CRLs made with lithium metal [1]. We report here detailed characterization of the response and resolution of the imaging system when using a thinly doped or a thick YAG:Ce screen. We provide also an example of its application to test the focusing properties of a new 2D Li multiprism lens [1].

The experiments were performed at beamline 7ID of the Advanced Photon Source. For this experiment, the monochromator was set to $10.0 \mathrm{keV}$, and the second monochromator crystal was detuned to cut the intensity by $50 \%$ [4]. The detuning reduced the contamination from higher harmonics. An ion chamber recorded the intensity before the camera, and a Roper CoolSNAP-cf camera equipped with a microscope with X2, X5, or X10 objectives [5] imaged the visible fluorescence by a YAG:Ce single crystal. Figure 1A shows the CCD camera, microscope, 90 degree mirror and YAG:Ce screen, as well as its mechanical support. A computer-controlled stage allowed us to focus the microscope on the screen by changing the working distance between the screen and the objective.

Figure 1B shows the detector response with the thick $0.5 \mathrm{~mm}$ YAG:Ce crystal. The incident flux was calculated from the ion chamber signal. Because the monochromator was detuned, little 3rd harmonic contamination was present. The foil transmission was consistent with values from tables. The three curves shown in the figure are consistent with each other. Small differences are caused by slight background drifts. Note that the zig-zag in the linear plot is caused by large background fluctuations of the CCD readout noise. This source of noise was attributed after the experiment to a bad A/D board and has since been fixed. This camera now has a root-mean-square (RMS) read-out noise of about 1.5 analog to digital units (ADU). The linearity of the CCD is fairly good, although upon detailed inspection, one would notice evidence of nonlinear behavior. This is expected since the manufacturer specifies a linearity better than 5\%.

Linear fits of these response curves were performed for two screens: a thinly doped ( $4 \mu \mathrm{m}$ Ce doping) YAG:Ce screen [6] and a single-crystal YAG:Ce screen $0.5 \mathrm{~mm}$ thick [7]. The thick YAG response is $3.17 \times 10^{-3}$ ADU/10 $\mathrm{keV}$ photons. When the doped side is facing the incident X-rays, the thin YAG response is $2.50 \times 10^{-4} \mathrm{ADU} / 10 \mathrm{keV}$ photons; while when the undoped side faces the incoming X-ray beam, it is $1.98 \times 10^{-4} \mathrm{ADU} / 10 \mathrm{keV}$ photons. The thick YAG response is 12.7 times larger than the thin YAG response. If only a thin layer of $4 \mu \mathrm{m}$ is doped, this thin layer absorbs only $(1-\exp (-4 / 57.1)) \approx 0.07$ [8]. This absorption is quite consistent with the measured ratio between the response of the thick and thin YAG:Ce screens. The thin YAG provides 1.26 times more light when the doping faces the incoming $\mathrm{X}$-rays than when the undoped surface faces the X-rays. This is surprising since one would expect that the transmission of the undoped YAG substrate $186 \mu \mathrm{m}$ thick would be $\exp (-186 / 57.1) \approx 4 \%$ [8]; thus the response 

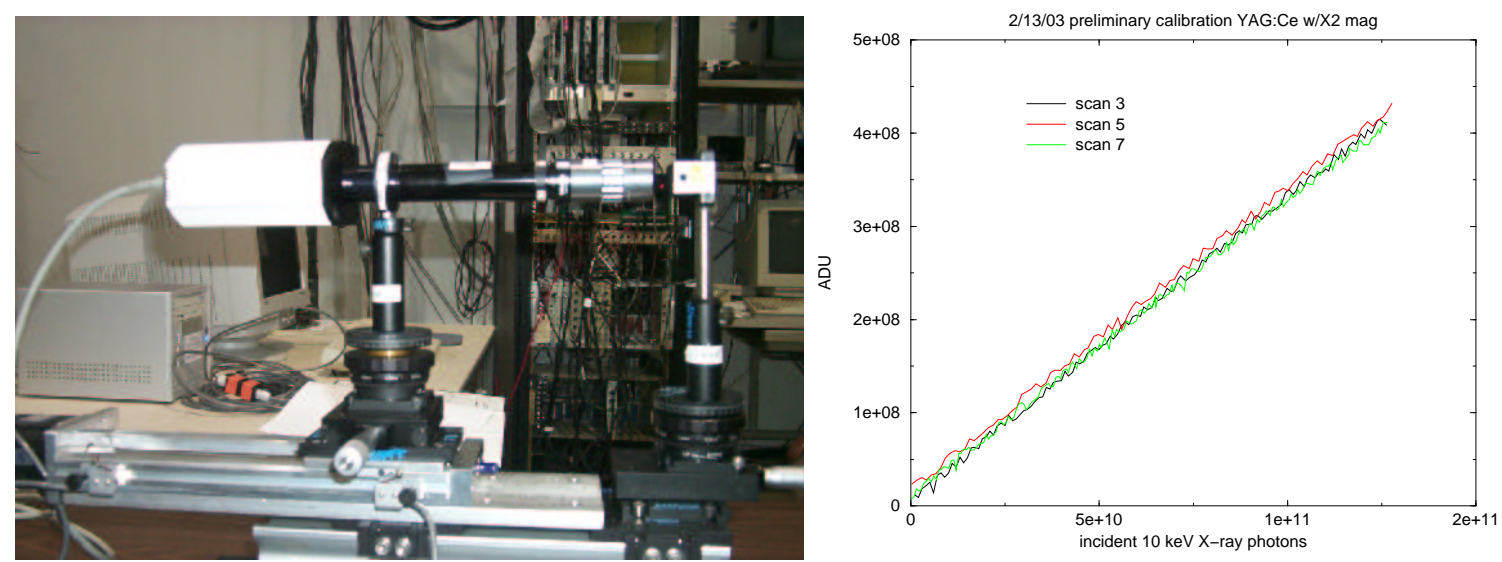

FIGURE 1. A) (left) The camera system. B) (right) The detector system response versus the incident number of photons per exposure time for the thick Yag:Ce crystal. Scan 3 had no Al filter in. Scan 5 and 7 used respectively a 0.18 and $0.36 \mathrm{~mm}$ thick foils.

should be quite different depending on the orientation. More work is needed to understand this unexpected response.

A YAG:Ce single crystal emits visible light at $550 \mathrm{~nm}$ when an X-ray is absorbed. The CCD quantum efficiency at this wavelength is about $35 \%$. Note that $1 \mathrm{ADU}$ is $3 e$ collected in the CCD well. When a $10 \mathrm{keV} \mathrm{X-ray} \mathrm{is} \mathrm{absorbed} \mathrm{in}$ YAG:Ce, 311 visible photons (v-ph) are emitted in $4 \pi$ [3]. The microscope collection efficiency is $C E=0.25(N A / n)^{2}$, where $\mathrm{NA}=0.055$ is the numerical aperture of the $\mathrm{X} 2$ objective, and $\mathrm{n}=1.95$ is the index of refraction of the YAG screen [3]. Given that $C E=1.99 \times 10^{-4}$ here, we expect $0.062 \mathrm{v}$-ph per $10 \mathrm{keV} \mathrm{X-rays} \mathrm{absorbed,} \mathrm{focused} \mathrm{on} \mathrm{the} \mathrm{CCD} \mathrm{plane.}$ Thirty-five percent of these photons are detected and collected as electrons in the CCD well. The scale factor for the thick YAG is expected to be $(0.062 \mathrm{v}-\mathrm{ph}) *(0.35 \mathrm{e} / \mathrm{v}-\mathrm{ph}) *(1 \mathrm{ADC} / 3 \mathrm{e})=0.0072 \mathrm{ADC} / 10 \mathrm{keV}$ photons. The calibration for the thick YAG is a factor of 2 off, but, given the approximate flux calibration, the agreement seems reasonable. Furthermore, reflection losses at several interfaces have been ignored, such as the YAG to air interface (10\%), mirror interface $(\approx 10 \%)$, in the objective, and in the tube lens.

To test the resolution of the camera, an edge was placed in the beam about $2 \mathrm{~cm}$ in front of the YAG crystal. A cleaved piece of a GaAs (100) wafer, $300 \mu$ m thick was used. It is well known that GaAs cleaves with atomically flat edges. Figure 2A shows a typical slice of the CCD image where the GaAs edge blocks the beam. The data were fit to an arctangent function with the intensity

$$
I=A \times \arctan \left(\left(x-x_{0}\right) / W\right)+B
$$

where $A$ and $B$ are constant, $x_{0}$, is the center of the edge, and $W$ is the width.

Figure 2B shows the widths from the least squares fit of a slice of the CCD data. The width is plotted versus the working distance between the microscope and the YAG:Ce surface. The solid line is a least square fit with

$$
W=W_{\min }+C\left(X-X_{c}\right)^{2},
$$

where $X_{c}$ is the CCD position at the best focus, $C$ is a proportionality constant, and $W_{\min }$ the best focus resolution. In Figure $2 \mathrm{~B}$, the best resolution is $W_{\min }=0.61$ pixels or $1.4 \mu \mathrm{m}$. If one calls the depth of focus (DOF), the distance where $W=2 W_{\text {min }}$, then replacing in Eq. 1, one finds that

$$
D O F=\left|X-X_{c}\right|=\sqrt{W_{\text {min }} / C} .
$$

In Figure 2B the DOF is $0.18 \mathrm{~mm}$. From the objective's specifications, one would have expected $0.09 \mathrm{~mm}$; thus the difference is likely caused by a different definition for the depth of field.

Similar results were obtained for different YAG crystal screens and visible optics setup. They are displayed in Table 1. Two different distances between the tube lens and the CCD were used: 142.5 and $141 \mathrm{~mm}$. The resolution is best with the tube length of $142.5 \mathrm{~mm}$ [5](compare 1 and 3). As expected the thin YAG works best when the X-rays are incident on the doped side (compare 4 and 5). Surprisingly, the single-crystal YAG:Ce performs much better than its thin counterpart even for magnifications as large as X10. The thick YAG was used for the focusing tests in Figure 3 

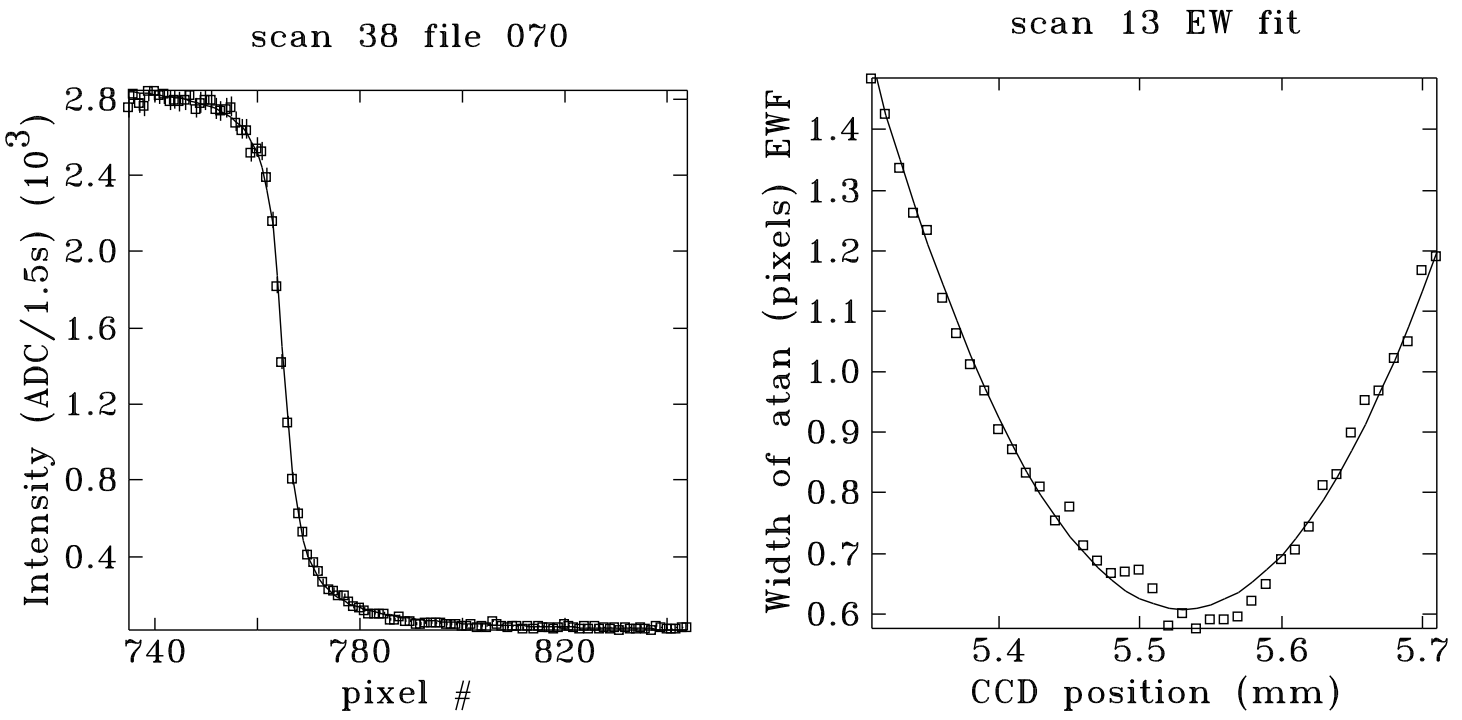

FIGURE 2. A)(left)The edge profile measured with a thinly doped $4 \mu \mathrm{m}$ YAG:Ce single crystal with the doping facing towards the incoming X-rays. B)(right) The fitted width $\mathrm{W}$ versus the working distance of the X2 objective from a thick YAG:Ce doped screen.

TABLE 1. Best resolution and DOF for several experimental conditions. The energy of the $\mathrm{X}$-ray beam used was typically around $10 \mathrm{keV}$. Eight separate measurements are labelled for ease of comparison. Some measurements were performed with a CoolSNAP HQ which has $6.45 / 4.65=39 \%$ larger pixels than the CoolSNAP-cf.

\begin{tabular}{llllll}
\hline Screen description & $\begin{array}{l}\mathrm{E} \\
(\mathrm{keV})\end{array}$ & $\begin{array}{l}\text { tube length } \\
(\mathrm{mm})\end{array}$ & Magnification & $\begin{array}{l}W_{\min } \\
(\mu \mathrm{m})\end{array}$ & $\begin{array}{l}\mathrm{DOF} \\
(\mathrm{mm})\end{array}$ \\
\hline 1-Thick YAG & 10.00 & 142.5 & 2 & 1.41 & 0.18 \\
2-Thick YAG & 10.87 & 142.5 & 5 & $2.02-2.95$ & 0.084 \\
3-Thick YAG & 10.00 & 141 & 2 & 1.91 & 0.22 \\
4-Thin YAG doped side & 10.00 & 141 & 2 & 5.32 & 0.61 \\
5-Thin YAG undoped side & 10.00 & 141 & 2 & 6.72 & 0.68 \\
6-Thin YAG doped side & 10.00 & 142.5 & 2 & 7.27 & $0.529^{*}$ \\
7-Thin YAG doped side & 10.87 & 142.5 & 5 & 6.01 & 0.175 \\
8-Thin YAG doped side & 10.00 & 142.5 & 10 & 2.51 & $0.042^{\dagger}$ \\
\hline
\end{tabular}

* Measured with a CoolSNAP HQ

$\dagger$ Measured with a CoolSNAP HQ

because its resolution is 2.8 times better than that for the thin YAG. The focus is much sharper, and the DOF is closer to the one specified by the objective's manufacturer. Taking the resolution as twice the fitted width, the best resolution achieved to date is $2.0 \times W_{\min }=2.8 \mu \mathrm{m}$.

We present next an example of the use of the detector system to image the focused and unfocused beam from novel lithium-based multiprism lenses. Two Li multiprism lenses mounted perpendicular to each other, with a $1 \mathrm{~mm}$ pitch, $111 \mathrm{~mm}$ length, and 90 degree teeth angle were used [1]. They were kept in a He-sealed tube with two Be windows [9]. The lens position and angle could be oriented by computer-controlled stages, with two spatial translation and two angular rotation stages. A slit placed in front of the lens reduced the beam size on the lens to $0.45 \mathrm{~mm}$ by 0.45 $\mathrm{mm}$. An ion chamber recorded the intensity after the lens. The source-lens distance was $51 \mathrm{~m}$, while the lens-camera distance was $4.5 \mathrm{~m}$. The demagnification is thus $11.3: 1$, and the focal length is $4.1 \mathrm{~m}$. The numerical aperture of the lens here is $0.11 \mathrm{mrad}$. Figure 3 shows slices of CCD images centered on the focused and unfocused beams. The focused beam vertical and horizontal FWHM are respectively 81 and $126 \mu \mathrm{m}$. The gain in Figure 3 is 12.6. Integrating the two raw images, in Figure 3, we find that the lens transmission is $82 \%$. This compares well with previous measurements on $\mathrm{Li}$ lens at $10 \mathrm{keV}$ and in similar geometries [1].

The imaging system presented here has been very versatile. We use it routinely to focus X-ray beams using 


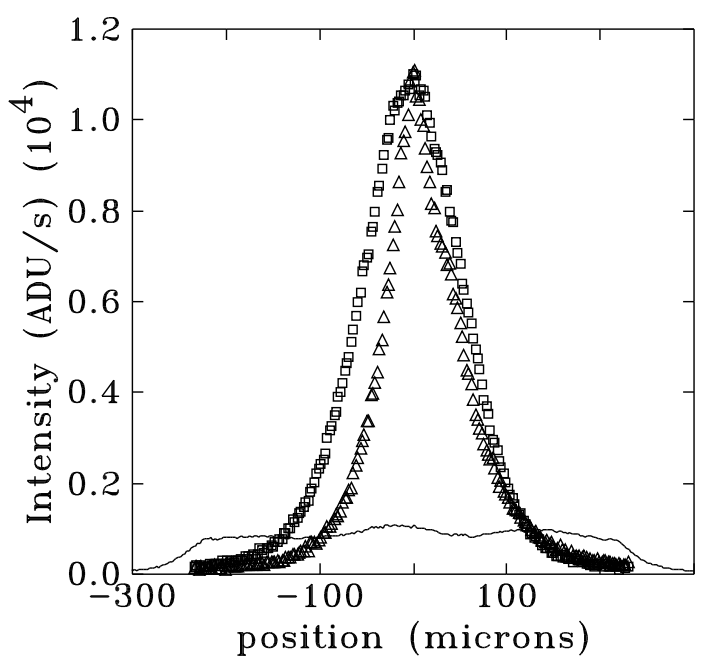

FIGURE 3. Horizontal slice of focused beam (squares). Vertical slice of focused beam (triangles). Vertical slice of the unfocused beam (solid).

Kirkpatrick-Baez mirror systems down to $1-2 \mu \mathrm{m}$. Because of the use of infinity-corrected objectives with a tube lens, one can put neutral density filters between the two lenses to attenuate the visible light, not the X-ray beam. Other options include placing $550 \mathrm{~nm}$ bandpass filter to reduce the background from other sources of light, or insert an iris to improve the resolution. This system has allowed us to quickly align Li lens in the beam significantly reducing the beam time required for the lens characterization.

\section{ACKNOWLEDGMENTS}

NRP's work is done with support from MDA through SBIR contract N00178-02-C-3119 and is monitored by the Naval Surface Warfare Center, Dahlgren Division. This work was done at the MHATT-CAT 7ID beamline, and was supported in part by DOE Grant No. DE-FG02-03ER46023 and DE-FG02-00ER15031, and by the NSF FOCUS physics frontier center. Use of the Advanced Photon Source is supported by the U.S. Department of Energy, Basic Energy Sciences, Office of Energy Research, under Contract No. W-31-109-ENG-38.

\section{REFERENCES}

1. Dufresne, E. M., Arms, D. A., Dierker, S. B., Clarke, R., Pereira, N. R., and Foster, D., Appl. Phys. Lett. 79,4085 (2001). Pereira, N. R., Arms, D. A., Clarke, R., Dierker, S. B., Dufresne, E. M., and Foster, D., Proc. SPIE 4502, 173 (2001). Arms,D. A., Dufresne, E. M., Dierker, S. B., Clarke, R., Pereira, N. R., and Foster, D., Rev. Sci. Instrum. 73, 1492 (2002).

2. Snigirev, A., Kohn, V., Snigireva, I., and Lengeler, B., Nature, 384, 49 (1996).

3. Kohn, A., Raven, C., Spanne, P., and Snigirev, A., J. Opt. Soc. Am. A, 15, No. 7, 1940 (1998).

4. Dufresne, E. M., Arms, D., Dierker, S.B., Clarke, R., Yacoby, Y., Pitney, J., MacHarrie, B., and Pindak, R., Rev. Sci. Instrum., 73, 1511-1513 (2002).

5. The objectives were the Mitutoyo MPlan APO long working distance infinity corrected objectives. The X2, X5 objectives were used here with the MT-40 tube lens (X1) refocusing on a C-mount CCD. Edmunds Scientific recommends in their catalog a C-mount tube length of $142.5 \mathrm{~mm}$.

6. 190- $\mu$ m-thick YAG, with 4- $\mu$ m-thick Ce doping purchased from St-Gobain Crystals and Detectors, 440-564-2251.

7. 500- $\mu$ m-thick YAG:Ce single crystal, purchased from www. startechinst.com, model F20G YAG:Ce doped.

8. Note that YAG has a composition of $\mathrm{Y}_{3} \mathrm{Al}_{5} \mathrm{O}_{12}$ with a density of $4.55 \mathrm{~g} / \mathrm{cm}^{3}$. Thus the X-ray absorption length at $10 \mathrm{keV}$ is $57.1 \mu \mathrm{m}$.

9. The two half lenses are housed in a stainless-steel pipe and sealed by two CF flanges each having a brazed in 0.125 -mm-thick Be window. The length of the unit is $403 \mathrm{~mm}$. If one removes the unit from the beam, the air transmission factor is 0.82 . 